\title{
Unusual manifestation of genital cutaneous leishmaniasis in an immunocompetent patient from São Paulo, Brazil: A case report
}

\author{
Luiza Campos Reis ${ }^{[1]}$, José Angelo Lauletta Lindoso[1],[2],[3] , Beatriz Julieta Celeste ${ }^{[1]}$, \\ Lucia Maria Almeida Braz ${ }^{[1]}$, Eduardo Milton Ramos-Sanchez ${ }^{[1],[4],}$ \\ Edite Hatsumi Yamashiro-Kanashiro[1], Hiro Goto ${ }^{[1],[5]}$ \\ and Luiza Keiko Matsuka Oyafuso[2]
}

\footnotetext{
[1]. Universidade de São Paulo, Faculdade de Medicina, Instituto de Medicina Tropical de São Paulo, São Paulo, SP, Brasil.

[2]. Secretaria de Saúde do Estado de São Paulo, Instituto de Infectologia Emílio Ribas, São Paulo, SP, Brasil.

[3]. Universidade de São Paulo, Faculdade de Medicina, Departamento de Moléstias Infecciosas e Parasitárias, São Paulo, SP, Brasil.

[4]. Universidad Nacional Toribio Rodriguez de Mendoza de Amazonas, Facultad de Ciencias de La Salud, Departamento de Salud Publica, Chachapoyas, Peru.

[5]. Universidade de São Paulo, Faculdade de Medicina, Departamento de Medicina Preventiva, São Paulo, SP, Brasil.
}

\begin{abstract}
A 31-year-old male patient developed an ulcer on the glans penis that evolved for three months without healing. We diagnosed it as leishmaniasis using polymerase chain reaction. No immunosuppression or associated diseases were observed. The patient was treated with meglumine antimoniate that cured the lesion in a month post-treatment. Here, we report this case of cutaneous leishmaniasis lesion at the unusual location of glans penis in an immunocompetent individual. The lesion likely developed due to the bite of a vector, highlighting the need for considering cutaneous leishmaniasis among differential diagnosis of sexually transmitted diseases in areas endemic for leishmaniasis.
\end{abstract}

Keywords: Cutaneous leishmaniasis. Diagnosis. Genital lesion.

\section{INTRODUCTION}

Cutaneous leishmaniasis (CL) is a vector-transmitted protozoan disease caused by different species of Leishmania. In Brazil, CL is mainly caused by the species Leishmania (Viannia) braziliensis, L. (V.) guyanensis, L. (Leishmania) amazonensis ${ }^{1}$, and approximately 238,000 cases of CL were reported between 2007 and 2018. In the state of São Paulo during the same period, 4,159 cases were reported, of which $55 \%$ were autochthonous cases ${ }^{2}$.

Its clinical manifestations vary from localized, disseminated, and diffuse cutaneous forms to severely mutilating mucocutaneous

\footnotetext{
Corresponding author: Prof. Dr. José Angelo Lauletta Lindoso.

e-mail: jlindoso@usp.br

(iD) https://orcid.org/0000-0002-4405-4401

Received 27 July 2020

Accepted 18 August 2020
}

lesions $^{1}$. The initial lesion is at the site of the vector bite in the form of a macule; after two weeks to three months, it may present as a small, pruritic, erythematous papule, and/or nodule and may involve draining lymph nodes. The lesion may resolve spontaneously or develop into a characteristic ulcer; some lesions may develop into other chronic forms. Mucocutaneous leishmaniasis is the most aggressive and mutilating form characterized by infiltration, ulceration, and destruction of mucous membranes and cartilage, and occurs years after the onset of CL. The lesions of CL are localized mainly in exposed body parts, such as the arm, leg, and face. Nasal and oral mucosae are the most affected in the mucocutaneous form. Its clinical manifestations depend on the species of the parasite, the vector, and the genetic and immunological constitution of the host ${ }^{1,3}$. The genital region is rarely a primary site. In immunosuppressed patients, however, studies have described genital involvement as a consequence of the spread of the parasite from the initial lesion ${ }^{4,5}$. 
The etiological diagnosis of CL is sometimes difficult, and therefore, epidemiological, clinical, and histopathological findings should be considered. Concerning clinical manifestations, the lesions present suggestive characteristics but may vary considerably. They may resemble ulcerated or non-ulcerated lesions from other diseases, such as Virchow's hanseniasis, paracoccidioidomycosis, tropical ulcers, syphilis, and cutaneous tuberculosis, thus requiring its differential diagnosis. Moreover, conventional diagnostic methods are limited in their efficiency, necessitating a combination of different approaches to diagnose leishmaniasis ${ }^{6}$.

Here, we report a case of CL with a lesion in the genital area in a patient from São Paulo, who did not exhibit any immunosuppression or associated disease, thus posing a challenge in diagnosis.

\section{CASE REPORT}

A 31-year-old male patient from Embu das Artes located in the municipality of the Metropolitan Region of São Paulo, in the state of São Paulo in Brazil sought an infectious disease consultation at the Instituto de Infectologia Emílio Ribas. He presented with an ulcer on the glans penis that evolved without healing for three months. The patient reported that he did not travel to other regions recently or perform outdoor activities and that he worked as a salesman in the city but not in rural areas. He had initially noticed a small lesion with raised border without pain that progressively increased in size over few weeks. Clinical examination revealed an ulcer with elevated borders and granulomatous contents (Figure 1A). The lesion measured $3.0 \times 2.5 \mathrm{~cm}$ in dermatological examination. It was not associated with systemic symptoms, such as fever, weight loss, hepatosplenomegaly, or lymphadenopathy. All hematological and biochemical parameters were within the normal range. Initially, the diagnosis was directed to sexually transmitted diseases, but tests for human immunodeficiency virus (HIV) and syphilis were nonreactive. Thus, we tested for CL based on the lesion characteristics and epidemiological data. Table 1 summarizes the tests performed for the diagnosis of CL - histopathology of the lesion biopsy, direct smear and culture in McNeal, Novy, Nicolle medium of the scraped lesion sample, polymerase chain reaction (PCR) of lesionderived DNA, and serological assays (anti-Leishmania enzyme-linked immunosorbent assay [ELISA] and indirect immunofluorescence [IIF]).

Histopathological examination revealed granulomatous dermatosis with diffuse pattern, vasculopathy, and evident plasmacytosis but Leishmania amastigotes. It was negative for acid-alcohol-resistant bacilli (BAAR) and fungi. We diagnosed and confirmed leishmaniasis based on the results of PCR for the $750 \mathrm{bp}$ kinetoplast DNA (kDNA) of the Leishmania subgenus (Viannia) (using the primers B1. 5'-GGGGTTGGTGTAATATAGTGG-3' and B2. 5'-CTAATTGTGCACGGGGAGG-3') (Figure 1B)', and the positive anti-Leishmania ELISA. The patient was treated with intravenous meglumine antimoniate $(20 \mathrm{mg} / \mathrm{kg} /$ day $)$ for 20 days. One-month post-treatment, the lesion completely healed (Figure 1C). The patient was followed up for six months with no

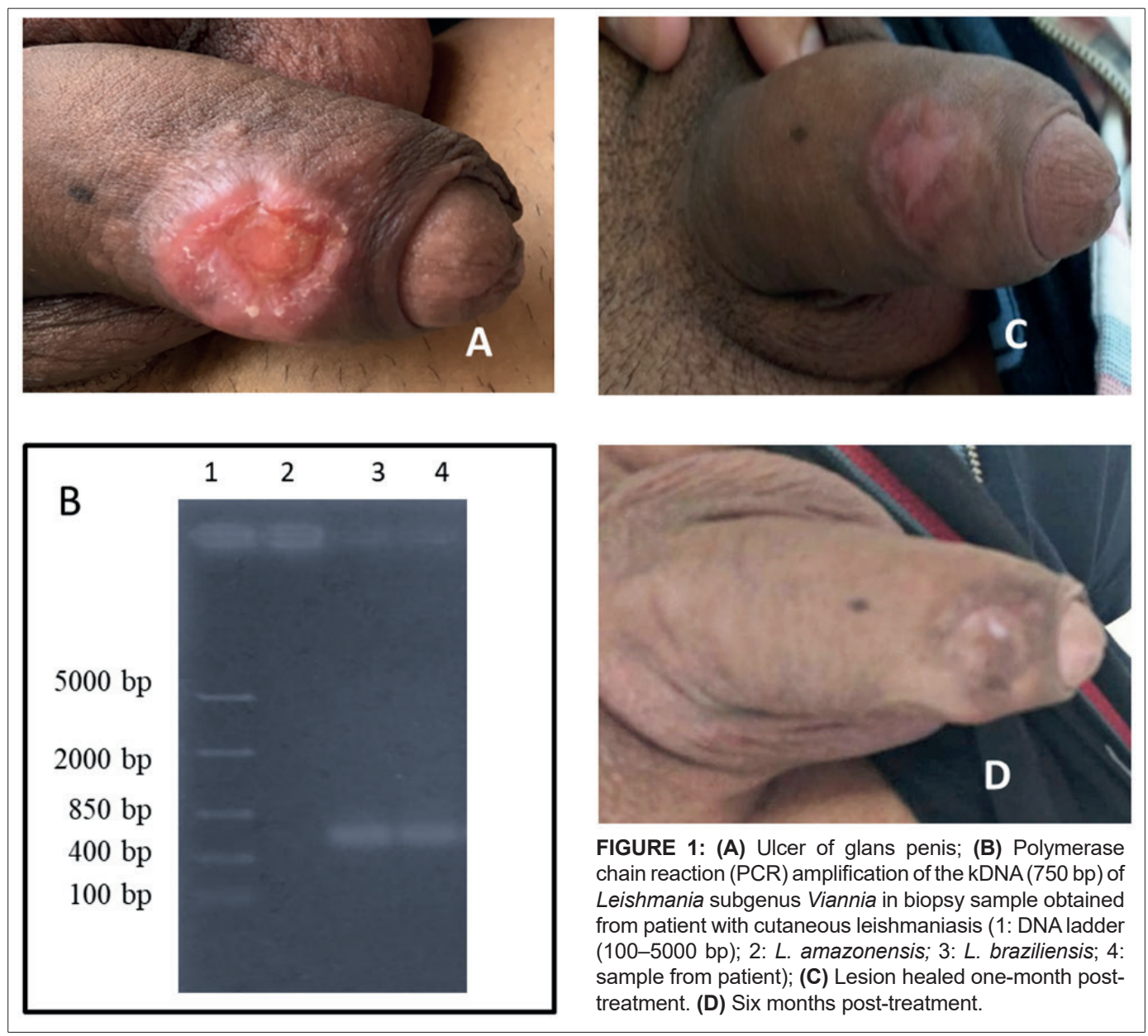


TABLE 1: Laboratory test results.

\begin{tabular}{lc}
\hline Diagnostic techniques & Results \\
\hline Direct smear & - \\
Parasite isolation & - \\
ELISA (IgG) & $+(1 / 160)$ \\
IIF (IgG) & - \\
PCR (kDNA) & + \\
\hline
\end{tabular}

ELISA: enzyme-linked immunosorbent assay; IIF: indirect immunofluorescence; PCR: polymerase chain reaction. +: positive; -: negative.

signs of relapse (Figure 1D) or any other lesion in other parts of the body and was considered cured. This study was approved by the Conselho Nacional de Ética em Pesquisa (National Council of Ethics on Research - CAAE: 71602417.0.0000.0065).

\section{DISCUSSION}

In $\mathrm{CL}$, the usual clinical presentation is a single cutaneous ulcerative lesion, usually localized in exposed areas of the body accessible to the sandfly ${ }^{8}$. Lesions of CL in the covered areas of the body is unusual; genital lesions are even rarer with only a few reported cases $^{4,5,8-11}$. Studies suggest that genital lesions may result from possible hematogenic dissemination in patients with multiple cutaneous lesions or in immunosuppressed patients $\mathrm{s}^{4,5,8}$. Our patient was immunocompetent and had just a single lesion with no history of CL.

Initially, the diagnosis was focused on sexually transmitted diseases; however, the results were negative for syphilis and HIV. The patient resides in a region with reported cases of leishmaniasis in the state of São Paulo, with the presence of the vector Lutzomyia intermedia, the main species related to the transmission of L. (V.) braziliensis ${ }^{12}$ in São Paulo. Based on this epidemiological analysis, the differential diagnosis for $\mathrm{CL}$ was considered. The diagnosis was confirmed using PCR for Leishmania kDNA and anti-Leishmania ELISA. Other parasitological tests (direct microscopic examination of sample aspirates and cultured material) for CL have low sensitivity, as parasites are few or absent in chronic lesions with long evolution periods ${ }^{1,6}$. Our results reinforce that CL should be diagnosed using a combination of clinical, epidemiological, and laboratory approaches, with more than one test performed. With accurate tracing of the patient's whereabouts, we concluded that the lesion on penis was not a secondary lesion but likely developed at the site of the vector bite when the patient reportedly slept naked due to hot weather; notably, this region is surrounded by the remnants of Atlantic forest.

The present case illustrates that in an area endemic for leishmaniasis, cases with atypical lesions should be investigated for leishmaniasis.

\section{FINANCIAL SUPPORT}

This work was supported by Fundação de Amparo à Pesquisa do Estado de São Paulo (fellowship 2019/25393-1 to LCR), the Conselho Nacional de Pesquisa (research fellowship to HG), LIM-49 and LIM 38 (Hospital das Clínicas, Faculdade de Medicina, Universidade de São Paulo).

\section{AUTHORS' CONTRIBUTION}

LCR contributed with data collection, data interpretation, literature search and writing; JALL contributed with patient clinical care, data collection, data interpretation, literature search and writing; BJC contributed with serological laboratorial test and data interpretation; LMAB contributed with molecular diagnosis and data interpretation; EMRS contributed with data collection, molecular diagnosis and writing; EHYK contributed with laboratorial tests and data interpretation; $\mathrm{HG}$ contributed with data interpretation, critical review and writing; LKMO contributed with patient clinical care, data collection and data interpretation.

\section{CONFLICT OF INTEREST}

The authors declare that there is no conflict of interest.

\section{REFERENCES}

1. Goto H, Lindoso JA. Current diagnosis and treatment of cutaneous and mucocutaneous leishmaniasis. Expert Rev Anti Infect Ther. 2010;8(4):419-33.

2. Ministério da Saúde (MS). Secretaria de Vigilância em Saúde. Departamento de Vigilância das Doenças Transmissíveis. Manual de Vigilância da Leishmaniose Tegumentar Americana. $1^{\mathrm{a}}$ edição. Brasília: MS; 2017. 189 p.

3. Garcia AL, Kindt A, Quispe-Tintaya KW, Bermudez H, Llanos A, Arevalo J, et al. American tegumentary leishmaniasis: antigen-gene polymorphism, taxonomy and clinical pleomorphism. Infect Genet Evol. 2005;5(2):109-16.

4. Guerra JAO, Coelho LIRC, Pereira FR, Siqueira AM, Ribeiro RL, Almeida THL et al. American tegumentary leishmaniasis and HIVAIDS association in a tertiary care center in the Brazilian Amazon. Am J Trop Med Hyg. 2011;85(3):524-7.

5. Lindoso JA, Barbosa RN, Posada-Vergara MP, Duarte MIS, Oyafuso LK, Amato VS, et al. Unusual manifestations of tegumentary leishmaniasis in AIDS patients from the New World. Br J Dermatology. 2009;160(2):311-8.

6. Vega-López F. Diagnosis of cutaneous leishmaniasis. Curr Opin Infect Dis. 2003;16(2):97-101.

7. De Bruijn MHL, Barker DC. Diagnosis of New World leishmaniasis: specific detection of species of the Leishmania braziliensis complex by amplification of kinetoplastid DNA. Acta Trop.1992;52(1):45-58.

8. Schubach A, Cuzzi-Maya T, Gonçalves-Costa SC, Pirmez C, OliveiraNeto MP. Leishmaniasis of glans penis. J Eur Acad Dermatol Venereol.1998;10(3):226-8.

9. Castro Coto A, Hidalgo Hidalgo H, Solano Aguilar E, Coto Chacón F. Leishmaniasis of the genital organs. Med Cutan Ibero Lat Am. 1987;15(2):145-50.

10. Cabello I, Caraballo A, Millan Y. Leishmanisis in the genital area. Rev Inst Med Trop Sao Paulo. 2002;44(2):105-7.

11. Lyra MR, Pimentel MI, Madeira MF, Antonio LF, Lyra JPM, Fagundes A, et al. First report of cutaneous leishmaniasis caused by Leishmania (Leishmania) infantum chagasi in an urban area of Rio de Janeiro, Brazil. Rev Inst Med Trop Sao Paulo. 2015;57(5):451-4.

12. da Silva RA, Mercado VT, Henriques LF, Ciaravolo RMC, Wanderley DMV. Magnitude and trend of American Tegumentary Leishmaniasis in the State of Sao Paulo, Brazil, 1975 to 2008. Rev Bras Epidemiol. 2012;15(3):617-26. 